\title{
M-SEQUENCE GROUND-PENETRATING RADAR WITH NOVEL FRONT-END CONCEPT
}

\author{
Martin PEČOVSKÝ* ${ }^{*}$ Pavol GALAJDA*, Stanislav SLOVÁK* ${ }^{*}$, Miroslav SOKOL*, Martin KMEC ${ }^{* *}$ \\ *Department of Electronic Engineering and Multimedia Telecommunication, Faculty of Electrical Engineering and Informatics, \\ Technical University of Košice, Park Komenského 13, 04200 Košice, Slovak Republic, tel. +421 55602 4341, \\ E-mail: martin.pecovsky@tuke.sk \\ ** Departments of Electronic Measurement Research Lab \\ University of Technology, Max-Planck-Ring 14, 98693 Ilmenau, Germany
}

\begin{abstract}
This article deals with the ultra-wideband (UWB) front-end circuits for the ground penetrating radars (GPRs). New concepts for the GPR front-end circuits are proposed, which exploit electrically short active antennas to achieve minimal dimensions and the wide frequency band of the radar system as well as offer enhanced user experience in comparison to the currently used conventional GPR systems. This work suggests using the M-sequence radar as the core for the proposed GPR and sums up the specific issues, which have to be taken into account when designing the device transmitting radio waves through the inhomogeneous high-attenuation environment, typical for the GPR. Additionally, an overview of commercially available GPR antennas in the frequency band below $300 \mathrm{MHz}$ is introduced for the comparison with the proposed solution. The theoretical working principles are discussed and confirmed by the measurements to pave the way for the future research.
\end{abstract}

Keywords: ground penetrating radar, ultra-wideband, $M$-sequence, active antennas

\section{INTRODUCTION}

The development of the M-sequence ultra-wideband (UWB) radars has a long tradition at our department. In cooperation with our German partners Ilmenau University of Technology and Ilmsens, GmbH, we have been a part of exciting research in areas of data processing software as well as ultra-wideband hardware [1] and [2], enabling the M-sequence technology to become one of the standards for short-range sensing, often outperforming earlier approaches such as pulsed or frequency-modulated systems. With continuous research and consequential technological improvements, new applications of the Msequence radars are emerging. We can find these applications in a lot of areas. As an example we can introduce as follows: non-destructive testing [3], metrology [4], microwave imaging [5], quality control [6], medical engineering [7], localization and positioning [8], ambientassisted living and others [9]. One of such application which is also introduced in this article is the use of the Msequence radar for ground imaging [10] and [11], also known as ground penetrating radar (GPR). The growing interest in UWB techniques is also expressed in a number of international conferences and workshops such as the annual IEEE International Conference on Ubiquitous Wireless Broadband (former IEEE International Conference on Ultra-Wideband) and the bi-annual International Conference on Ground Penetrating Radar. However, the new applications require adaptation of the radar system to account for new deployment conditions. This is especially true for ground penetrating radars.

The term ground penetrating radar refers to the range of radar technologies used to detect and localize objects located under the ground surface or behind similar opaque structures exploiting reflection of electromagnetic waves. The first use of electromagnetic waves for this purpose was documented in 1910 in a German patent by Leimbach and
Löwy [12]. Along with other techniques, it has become a powerful tool for underground exploration nowadays.

Although intensive research attention has been payed to the GPR technology in the last decades, it still leaves a lot of research areas open because of its complexity and operation in a very harsh environment for radio wave propagation. The most challenging part of the groundpenetrating radar hardware is its front-end, i.e. antennas and analogue circuits connected directly to them and accounting for soil properties as well.

The electromagnetic media experienced in ground probing consists of an inhomogeneous mixture of materials featuring various electromagnetic properties. From the practical point of view, water content plays the significant role in the propagation of electromagnetic waves through the soil. Generally, soil attenuation grows rapidly with increasing frequency. This fact leads to the necessity to use low frequencies to achieve sufficient penetration depth, coming hand-in-hand with large antennas, impractical for handheld deployment. Moreover, the wide frequency band is required to achieve sufficient resolution of the radar image. Thus these features of the media under test require tight trade-offs in the GPR design.

In general, the challenges to the concurrent GPR technology can be summarized as follows:

- high attenuation of high frequency signals,

- extreme antenna size at low frequencies,

- large bandwidth required to achieve sufficient resolution,

- hard-to-predict inhomogeneous media under test (MUT),

- influence of MUT on front-end behavior.

To cope with the above mentioned GPR challenges, we suggest using the combination of three principles which is novel to GPR:

- M-sequence UWB technology,

- electrically short active antennas, 
- $\quad$ simultaneous measurement of voltage and current at the antenna.

This article deals with the overall system design of the M-sequence GPR operating in an unconventional 50 to $300 \mathrm{MHz}$ band equipped by novel transmitter and receiver front-ends. As the full-sized antenna would be extremely impractical in the required frequency band, electrically short active transmitting and receiving antennas were designed to solve this problem. By inserting the active transmitter and receiver circuit directly into the antenna feed point, matching of the short antenna to the feeder is overcome while eliminating feeder standing waves at once. Moreover, short antennas with active circuits allow for bringing the relatively simple theory of their operation to practice. In addition, miniaturizing the antenna dimensions makes its behavior more similar to the isotropic radiator, allowing to simplify localization algorithms [11]. By implementing the proposed principles, the M-sequence ground-penetrating radar may become a competitive replacement for pulse systems employed for ground scanning currently, which suffer from extreme peak pulse power required to transmit the signal through the highattenuation environment.

\section{M-SEQUENCE RADAR PRINCIPLES}

A general block diagram showing the working principle of the UWB M-sequence radar is shown in Fig. 1 [13]. A feedback shift register is clocked by a reference oscillator at a single tone $f_{c}$, to generate the M-sequence. More than $70 \%$ of the signal power is concentrated at frequencies below $f_{c} / 2$. Thus, limiting operation to frequencies below this value does not impair the performance significantly [13]. On the other hand, this restriction allows for sampling at $f_{c}$ without aliasing, as the Nyquist condition is kept. Because the $\mathrm{M}$-sequences are periodic, the $\mathrm{A} / \mathrm{D}$ conversion can be performed even at a lower rate given by the binary divider ratio. A complete set of data samples can be gathered during successive signal periods as long as the scene under test can be assumed to be time invariant [13]. The impulse response of the scene under test is calculated as the correlation between known transmitted M-sequence and received signal by the DSP block. More on the Msequence sensing principles can be found in [11].

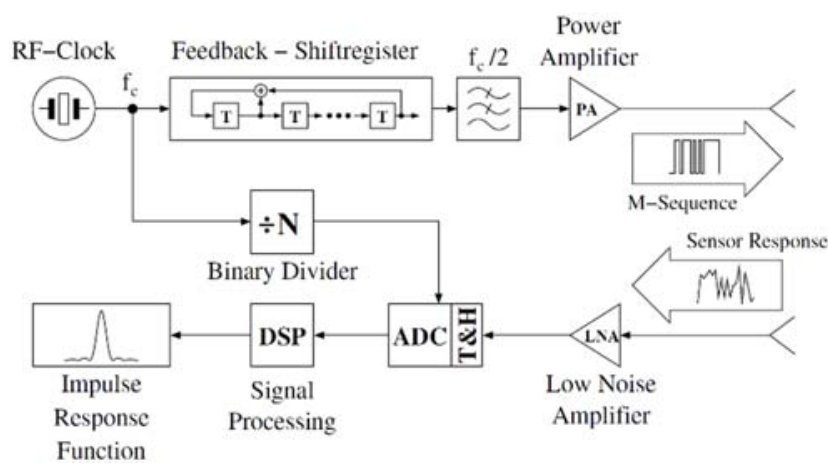

Fig. 1 M-sequence radar principle [13]

The main advantages of this concept are constant amplitude without peaks coming hand-in-hand with efficient time usage in terms of power, high immunity to interference and simplicity as well as versatility of the system which includes low number of analogue circuits. For example, the frequency band and therefore the resolution of the whole M-sequence radar can be set solely by the clock source frequency thanks to the wideband design of the device components in the form of application specific integrated circuits (ASICs). Ultra wide band of the M-sequence illumination signal allows to gather the high amount of the information about the medium under test, high resolution and wide range of applications. As opposed to pulsed systems most common for GPRs nowadays, the resolution is given by the bandwidth of the illumination signal rather than by the pulse width which is required to be extremely small for high resolutions. Moreover, the Msequence is transmitted continuously, overcoming the necessity to transmit high peak power pulses if large penetration depth in high attenuation media is required. If compared to frequency-modulated radars suggested for GPR purposes e.g. in [14] and [15], the M-sequence radar features simpler design with higher versatility and uses less components with critical tolerances. The previous work of the authors on the M-sequence GPR, concentrated on its receiving front-end, is documented in [16].

\section{M-SEQUENCE GROUND PENETRATING RADAR}

Each application of the M-sequence radar sensor concept sets specific requirements on its signal processing and especially on the analogue front-end. The main specific issues for the M-sequence GPR are:

- Upper frequency band limit is given by attenuation of high frequencies in the soil and required penetration depth. For the first proof of concept, the maximum operational frequency was chosen to be $300 \mathrm{MHz}$ while the M-sequence clock frequency is $1 \mathrm{GHz}$.

- Low frequencies have good ground penetration, however, low frequency limit is set by allowable antenna dimensions and its efficiency. Our first goal is the lower cut off frequency equal to $50 \mathrm{MHz}$.

- Hardware support for monitoring of variable antenna-to-ground coupling and electrical properties of the soil in the near field of the antenna is required for estimation of soil properties and improved antenna positioning [16].

The resulting M-sequence GPR frequency range (50 to $300 \mathrm{MHz}$ ) has a bandwidth of $250 \mathrm{MHz}$, which is much less than for typical short-range high resolution sensors. However, the ground medium has much higher permittivity compared to the air, therefore the GPR resolution is kept in reasonable range. The range resolution $\Delta r$ of the GPR for expected mean soil relative permittivity $\varepsilon_{r}=9$ can be calculated according to (1), where $c_{0}$ is the speed of light in vacuum and $B$ is the bandwidth of the illumination signal.

$\Delta r=\frac{c_{0} / \sqrt{\varepsilon_{r}}}{2 B}=\frac{3.10^{8} / \sqrt{9}}{2.250 .10^{6}}=0.2 \mathrm{~m}$ 
Table 1 Examples of GPR antennas for $50-300 \mathrm{MHz}$ band available on the market

\begin{tabular}{|l|c|c|c|c|}
\hline \multicolumn{1}{|c|}{ Manufacturer \& type } & $\begin{array}{c}\text { Center frequency } \\
\text { (MHz) }\end{array}$ & Bandwidth & Dimensions (cm) & Weight (kg) \\
\hline MALÅ GX160 HDR [17] & 160 & $120 \% @-10 \mathrm{~dB}$ & $72 \times 48 \times 19$ & 10,7 \\
\hline MALA GX80 HDR [17] & 80 & $120 \% @-10 \mathrm{~dB}$ & $101 \times 78 \times 22$ & 24,6 \\
\hline Mala [18] & 200 & $100 \%$ & $46 \times 26 \times 3$ & 4,2 \\
\hline GSSI 50270S [19] & 270 & & $45 \times 45 \times 17$ & 8,6 \\
\hline GSSI 5106A [19] & 200 & & $60 \times 60 \times 30$ & 20,5 \\
\hline GSSI 3207AP [19] & 100 & & $25 \times 96 \times 56$ & 13 \\
\hline GSSI MLF3200 [19] & 15 to 80(variable) & & $184 \times 14 \times 5$ & 15 to 23 \\
\hline Sensoft pulseEKKO [20] & 50 & & $92 \times 14 \times 5$ & 1,8 \\
\hline Sensoft pulseEKKO [20] & 100 & & $46 \times 14 \times 5$ & 0,8 \\
\hline Sensoft pulseEKKO [20] & 200 & & $96 \times 76 \times 17$ & 9,5 \\
\hline Sensoft Noggin 100 [21] & 100 & $100 \% @-3 \mathrm{~dB}$ & & \\
\hline
\end{tabular}

The resulting range resolution of $20 \mathrm{~cm}$ is sufficient for applications in archeological research, which should be the first application of the device. Bandwidth and bandwidthdependent resolution may be later adjusted by increasing system clock frequency and adapting filters and front-end circuits for new requirements.

As the frequency range of the GPR covers significantly lower frequencies than typical $\mathrm{M}$-sequence radars designed for other purposes (mostly in FCC or ECC band from 3 to $10 \mathrm{GHz}$ ), antennas and front-end analogue circuits have to be redesigned. Thanks to the $\mathrm{M}$-sequence constant amplitude, requirements on the dynamic range of the analogue circuits are much more relaxed than of those for pulse-based GPRs [16]. Moreover, the M-sequence is a rectangular-like signal, therefore the linearity requirements are not that strict as well.

\section{STATE OF THE ART GPR ANTENNAS}

Selected properties of the practical GPR antennas available on market nowadays are summed up in Table 1.

Many of the antennas are sold together with the GPR unit, sometimes in the active antenna configuration, i.e. front-end electronics are placed in the same housing with the antenna. Unfortunately, GPR manufacturers mostly describe their products as black boxes with more or less specified properties, without providing any information about their working principles. Therefore, it is difficult to compare various products without knowledge of the technology used and often even without test conditions specified. However, by keeping the technological details secret, the manufacturers give us an obvious signal, that the research in the GPR area is still ongoing and new techniques can still be introduced.

In Table 1, frequency range, dimensions and weight of the GPR antennas are given as stated by the manufacturer. The list is non-exhaustive and is focused on antennas in 50 to $300 \mathrm{MHz}$ range, for which the solution will be proposed in the next section. Although information about the antenna bandwidth is not exactly specified in the most of the antenna datasheets, we may assume it to be about $100 \%$ of the center frequency. Looking at Table 1, it is obvious that there is no antenna covering the whole range from 50 to
$300 \mathrm{MHz}$ and mechanical antenna exchange is required to cover more than approximately $100 \%$ bandwidth. Moreover, antennas with the center frequency lower than $100 \mathrm{MHz}$ have large dimensions unsuitable for portable equipment.

\section{DESIGN OF THE GPR FRONT-END}

The simplified block diagram of the proposed Msequence GPR is shown in Fig. 2. The GPR consists of two measurement heads denoted as master and slave due to the clock synchronization architecture. The master includes the clock generator and features large current radiator frontend, optimized for transmitting. On the other hand, the slave device is driven by the clock signal from the master and is equipped with a short dipole antenna optimized for the reception. Therefore, the master and slave together create a bi-static short-range radar with master sending the M-sequence signal of sufficient power and slave performing as the receiver by measuring the voltage at the short dipole antenna in the basic mode of operation. However, the proposed system has the second mode of operation which can be periodically switched with the first one, called the coupling measurement mode. In this mode, both antennas are allowed to transmit (simultaneously or sequentially, dependent on the operator's requirements) while voltage and current at the antennas are measured. In this way, properties of the antennas are monitored including the coupling of the antennas to the ground, and electrical properties of the material in the antenna near-field may be estimated. This mode is especially useful for the calibration of the radar signal processing and the adjustment of antenna placement on the ground surface.

The radar core in both heads consisting of clock distribution circuits (clock source, buffers and frequency divider by 64), an M-sequence generator and an ADC with FPGA control and the preprocessing unit is based on the typical M-sequence design discussed earlier and shown in Fig. 1. Front-end circuits drive the proposed antenna elements with the $\mathrm{M}$-sequence signal and allow for measurement of current and voltage directly at antenna feedpoints. The architecture of the front-end circuits and antennas will be discussed in details in further subsections. 


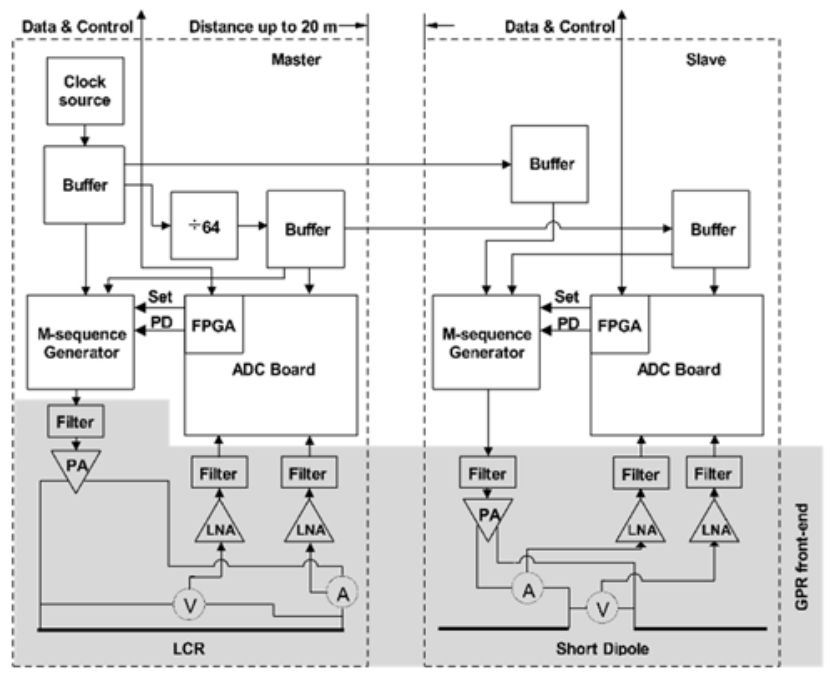

Fig. 2 Block diagram of the proposed M-sequence GPR

\subsection{Short dipole front-end}

The short dipole is a well-known antenna featuring typically high input impedance consisting of very low radiation resistance and capacitive reactance. The radiation resistance is typically well below $10 \Omega$ and grows quadratically with the dipole length. Omitting the antenna losses and low radiation resistance, the dipole can be modeled by a capacitor $C_{d}$ loading the transmitter. If considered as a receiving antenna, we can imagine the short dipole as an AC voltage source $V_{0}$ with series capacitance $C_{d}$ (Fig. 3). The open-circuit voltage $V_{0}$ on the short dipole is given by

$V_{o}=E \cdot L_{e f f}$

where $L_{\text {eff }}$ is the effective length of the dipole dependent on its physical dimensions and $E$ is the magnitude of electric field intensity tangent to the dipole [22]. Equation (2) shows that the amplitude of the voltage induced in the unloaded short dipole is independent on frequency. From this point of view, the most important property of the active circuit designed for receiving active antenna is its input impedance which loads the dipole. Its importance may be deduced from Fig. 3.

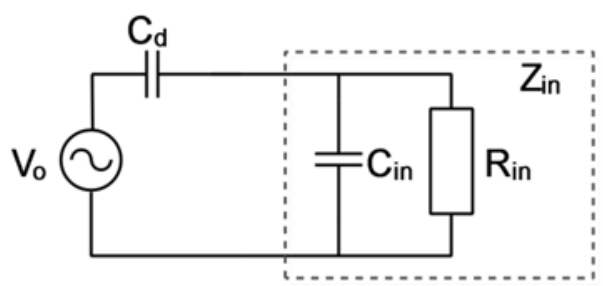

Fig. 3 Equivalent circuit of the short dipole loaded by the amplifier input

The voltage source $V_{0}$ and capacitor $C_{d}$ form the short dipole equivalent circuit. The input impedance of the amplifier $Z_{\text {in }}$ connected directly to the feed point of the dipole is modeled as a parallel combination of resistance $R_{i n}$ and capacity $C_{i n}$. It is obvious that although the input capacity of the amplifier forms together with $C_{d}$ a frequency-independent AC voltage divider, the input resistance $R_{i n}$ creates a high-pass filter attenuating the received signal at low frequencies. In ideal case, with $R_{\text {in }}$ approaching infinity, we get frequency independent active antenna. Therefore, we conclude that with the proper design of the active element for the short dipole antenna, it is possible to obtain a flat frequency response over a wide band of frequencies even if the antenna dimensions are much smaller compared to the wavelength.

The receive $(\mathrm{Rx})$ amplifier with high input impedance was constructed of ultra-fast operational amplifiers placed as close as possible to the center of the dipole. The details of the amplifier design are elaborated in [16] as well as the detailed description of the short dipole UWB receiving antenna.

In addition to reception of the signal radiated into the MUT by the master head of the proposed GPR, the short dipole front-end should be able to measure the antenna to ground coupling as well. That is accomplished by the measurement of voltage and current at the antenna while it is excited by the M-sequence signal. The corresponding front-end circuit was designed in [23].

Exploiting these principles, planar short dipole active antenna with dimensions of $20 \times 20 \mathrm{~cm}$ was constructed for reception in 50 to $300 \mathrm{MHz}$ band.

\subsection{Large current radiator front-end}

The large current radiator (LCR) was pioneered by $\mathrm{H}$. F. Harmuth in the 1980s [24]. It is derived from Hertzian dipole as described in Fig. 4. Let us consider the Hertzian dipole, i.e. very short dipole with capacitive terminations on both ends (Fig. 4a.). The Hertzian dipole is fed by an alternating current which flows through the dipole arms into the capacitive terminations while radiating electromagnetic waves proportional to the first time derivative of the current. In practice, it is difficult to realize such antenna efficiently because of extremely high voltage or capacitance necessary to excite sufficient current in the dipole. This may be overcome by simply interconnecting the ends of the dipole arms by the conductor (Fig. 4b.). The charge carried by the current does not have to be stored in the terminations anymore, but has a free path in the loop.

Nevertheless, the current of the same magnitude but the direction opposite to the dipole current flows through the conductor which connects the dipole ends. This return current radiates electromagnetic waves with opposite direction of electric field vector. Radiated fields interfere and result in weak radiation proportional to the second time derivative of the dipole current [25].

The cancellation of the electromagnetic fields may be avoided by a plate made of absorbing material inserted between the dipole and return conductors to separate their half-spaces on the cost of absorbing a part of radiated energy. Work [25] describes using a metal plate covered with a material with permeability around 10 and sufficient ohmic loss as the most convenient solution. Propagation around the plate has to be avoided as well. 
a.)

b.)

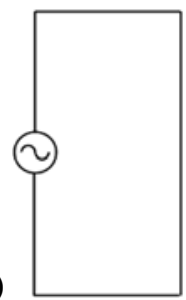

c.)

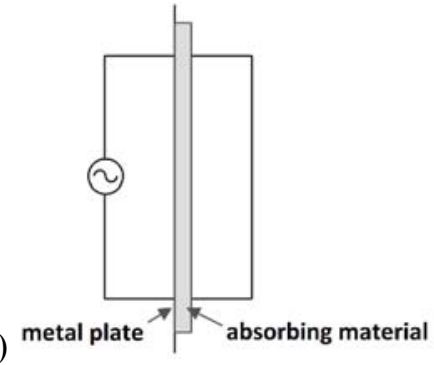

Fig. 4 LCR principles. a) Hertzian dipole, b) short-circuited Hertzian dipole, c) LCR setup

The role of the LCR front-end circuits is to drive sufficient current of the appropriate waveform through the radiator as well as to measure the voltage and current at the antenna for ground coupling and soil material properties estimation. As opposed to the short dipole front-end, the LCR setup is optimized for transmission.

To achieve good low-frequency performance, the LCR current should be the time integral of the M-sequence. Then, the radiator performs a single time-derivative and the resulting electrical field has a similar spectrum as the original $\mathrm{M}$-sequence signal. There are basically two approaches to excite the LCR by the M-sequence signal:

- voltage source with signal integration on the LCR inductance

- current source with signal integration on a low pass filter

The voltage source driver is easily implementable e.g. by an emitter follower stage, but it suffers from changes of antenna parameters and low efficiency. On the other hand, the current source driver approach should bring improvements at a cost of more complicated circuit design. The state of the art LCR drivers are summarized in [23] and a driver for GPR applications is proposed in [16]. However, the research is still ongoing.

\section{SIMULATIONS AND MEASUREMENTS}

To confirm the above discussed proposals, simulations were executed, consequently the prototypes were manufactured and measured. A brief overview of the obtained results will be presented in this section.

First of all, the behavior of the short planar bowtie antenna with dimensions $15 \times 15 \mathrm{~cm}$ was simulated in the presence of soil material. In Fig. 5, the radiation patterns in $\mathrm{E}$ and $\mathrm{H}$ plane are shown for soil permittivity $\varepsilon_{r}=9$. From the figure, it is obvious, that no bulky directional antenna is needed to achieve reasonable directivity because the electromagnetic field is coupled mostly into the medium with higher permittivity if the antenna is placed on the airground interface. This confirms the assumption that an electrically short dipole-like antenna is a good choice for GPR applications regardless of its omnidirectional radiation in free space.
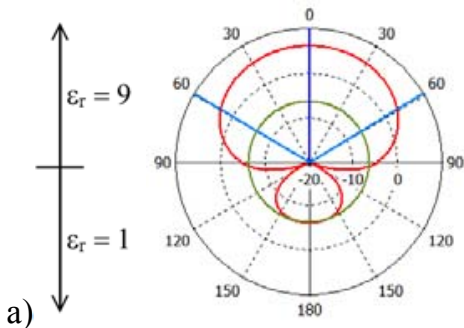

b)

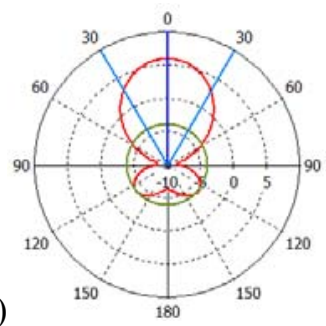

Fig. 5 Radiation patterns of the short $15 \times 15 \mathrm{~cm}$ bowtie placed on the ground surface with $\varepsilon_{r}=9$ at $100 \mathrm{MHz}$ : a) E-plane, b) H-plane

Secondly, a mathematical simulation of the voltagesource LCR excitation has been performed. The results are shown in Fig. 6. The results prove that if the LCR current is the integral of the original M-sequence source voltage, the spectrum of the radiated electric field is similar to the original M-sequence spectrum.

After the simulations were performed, the first prototypes have been built and tested. The photo of the manufactured electrically short dipole antenna with frontend circuits placed at its feedpoint is shown in Fig. 7.

A prototype of the LCR front-end was manufactured as well. However, it will not be discussed in details here because it may be subject to patent application in the future.

The active short dipole antenna in Fig. 7 was compared to the same-sized passive bowtie in the receiving mode placed in the same distance from another dipole transmitting antenna. The spectrum of the received signals as measured by the M-sequence radar is shown in Fig. 8.

From the Fig. 8 it is clear, that the proposed active antenna has a significantly better performance at low frequencies compared to the passive dipole. The lower cutoff frequency for $10 \mathrm{~dB}$ drop moved from $163 \mathrm{MHz}$ with the passive antenna to $95 \mathrm{MHz}$ for the proposed active antenna. Since the measurements were taken in an open air environment, further equalization of the spectra is assumed because of stronger attenuation of high frequencies in the soil. Therefore, $10 \mathrm{~dB}$ lower cut-off frequency is expected to be below $50 \mathrm{MHz}$. Upper cut-off frequency $320 \mathrm{MHz}$ is given by a low-pass antialiasing filter placed into the receiving signal path and can be increased up to $500 \mathrm{MHz}$. Steep drops in the received signal spectra are mostly caused by multi-path reflections inside the room, where the measurements were taken. Measurements in an anechoic chamber would give more precise results. Unfortunately, the anechoic chamber was not available to the authors during the creation of this paper. Simple gating of multipath signals was not sufficient in this case because of the long sampling period of the received signal if compared to a typical multipath time delay experienced in the indoor environment. However, the signal received by the passive antenna shows similar behavior, therefore we can exclude that it originates in the proposed circuitry.

The amplitude is given in $\mathrm{dB}$ relative to the maximum value for better bandwidth comparison in Fig. 8. Actual gain of the proposed active antenna above the passive dipole is about $5 \mathrm{~dB}$ higher than displayed. 

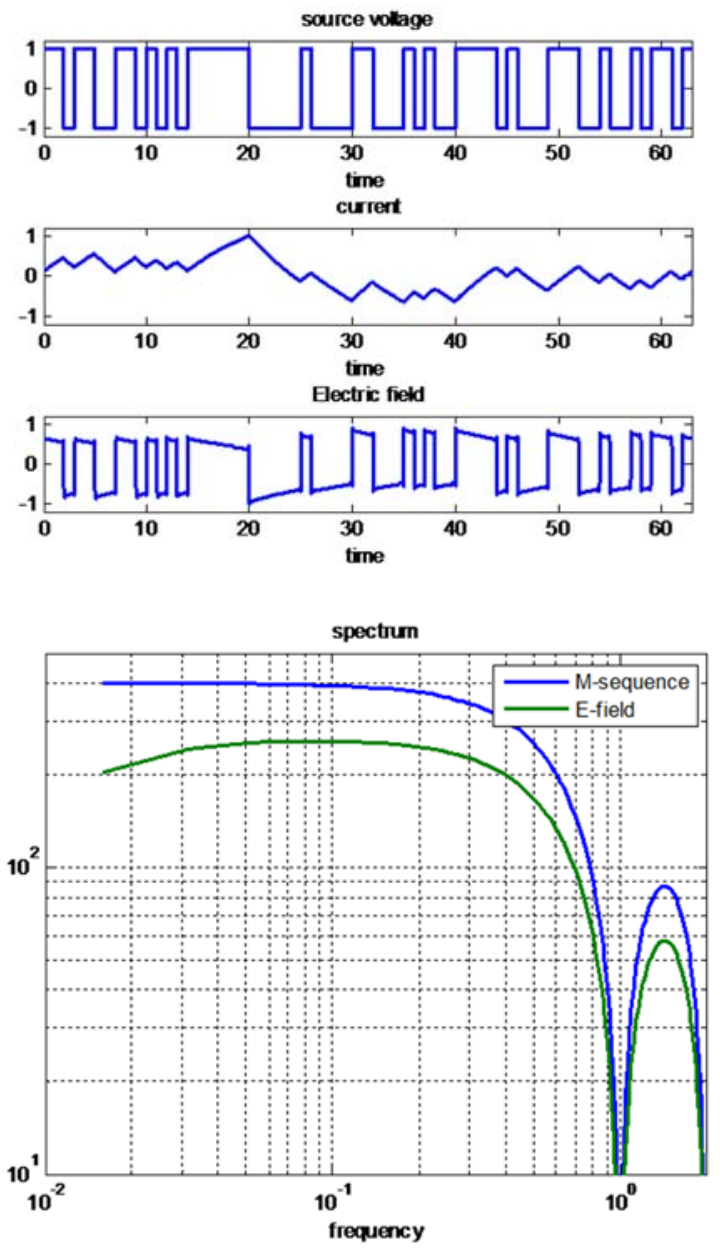

Fig. 6 Mathematical simulation of the LCR operation

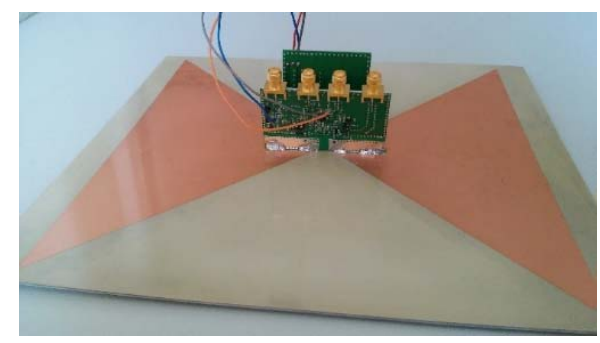

Fig. 7 Prototype of the short-dipole GPR front-end

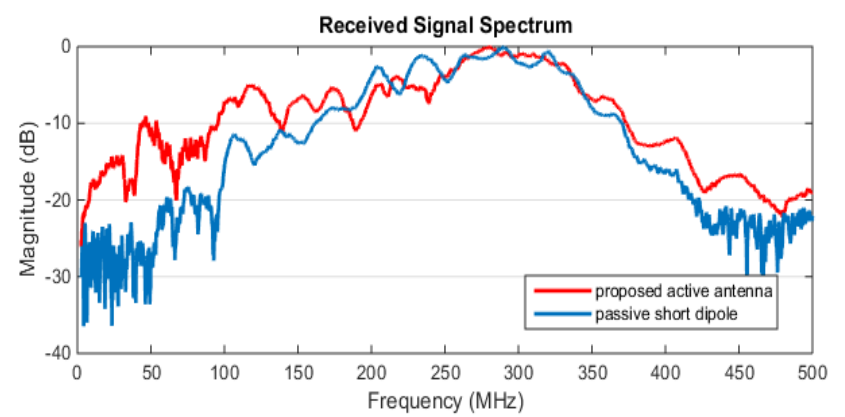

Fig. 8 Comparison of the received signal spectra of the proposed active antenna and equally sized passive dipole.

To find out if the electrically short active antennas introduced in this article improve the GPR system lowfrequency performance, spectra of the received signals were calculated from the in-system measured impulse responses. For comparison with a reference full-sized antenna, one of the designed antennas (active LCR at Tx or dipole with the active circuit at $\mathrm{Rx}$ ) was replaced by a conventional reference antenna. Comparison of the received spectra with a conventional reference antenna at the transmitting (blue) and receiving (green) side as well as complete transmission chain of the proposed system prototype (red) is shown in Fig. 9. As the reference antenna, a double rigged waveguide horn antenna DRH200 [26] was used.

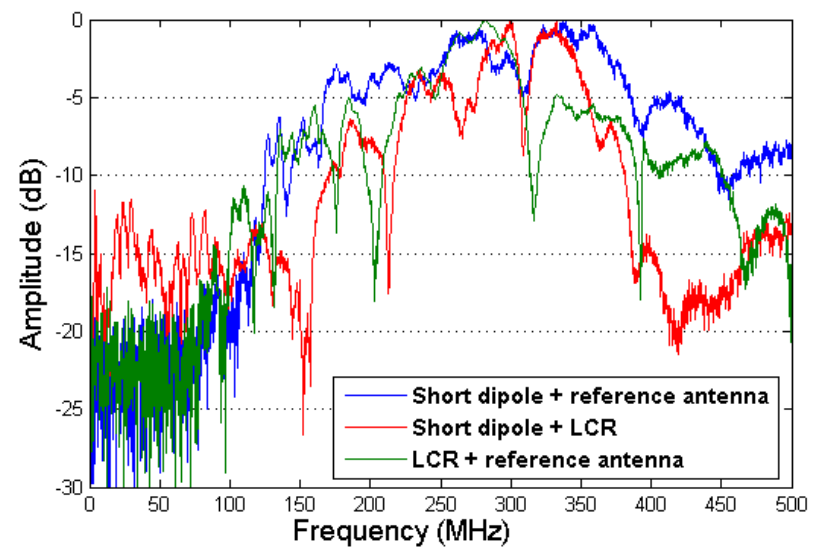

Fig. 9 Comparison of the in-system measured received spectra

The relative amplitude of the received signal is approximately $8 \mathrm{~dB}$ higher at frequencies below $100 \mathrm{MHz}$ when using solely the active antennas described in this article, as proven by measurement results shown in Fig. 9 . The loss of high frequencies is given by maximum operating frequency of the proposed active circuits and may be improved by using components suitable for higher frequencies, if needed. However, the high frequencies are of less interest in the considered GPR application, as they are attenuated by the soil. The deep drop in the spectrum in Fig. 9 around $150 \mathrm{MHz}$ is probably caused by multipath propagation of the signal in the indoor environment which becomes more significant with the proposed miniaturized antennas than with the directional reference antenna. It should not appear if the measurement was taken in an anechoic chamber.

From the results, we may assume that the concept of electrically short active antennas brings improvement to the low-frequency performance of the GPR M-sequence radar. While reducing the size (both master and slave measurement heads of the proposed M-sequence GPR have overall dimensions approx. $30 \times 30 \times 10 \mathrm{~cm}$, whereas the reference horn antenna without electronics has dimensions about $100 \times 100 \times 80 \mathrm{~cm}$ ), and costs of the antennas, at the same time with the low-frequency gain increase, the results may be considered as a good starting point for the further research.

\section{CONCLUSION}

In this paper, we have shown that the main hardware GPR challenges are miniaturizing of antennas dimensions and improvement of their wideband performance for frequencies below $300 \mathrm{MHz}$, where larger penetration depths are achievable. Additionally, the refined design of the GPR front-ends including active antennas should result 
in elimination of the need to deal with devices of up to several meters in length.

For the accomplishment of this task, the concept of electrically small active antennas was chosen and principles of the M-sequence radars have been implemented for the ground scanning applications. Design of the antennas not apart from, but together with the whole radar front-end, its electronics as well as the device mechanical setup, brings a new degree of freedom to the system designer. In this work the novel design of front-ends was proposed. Both front-ends exploit simple antenna elements together with the active circuits designed to match the parameters of the antennas. Therefore, lower cut-off frequency of the device is reduced and small antenna dimensions are achieved at the same time. Moreover, the M-sequence radar principle is proposed for the GPR instead of the common pulse radar. The main advantages are the constant amplitude of the illuminating signal, high variability of the radar properties and low costs if the mass production of application-specific integrated circuits will be launched.

\section{ACKNOWLEDGMENTS}

This work was supported by the Slovak Research and Development Agency under the contract No. APVV-150692, the Scientific Grant Agency (VEGA) under the contract No. 1/0772/17 and the Slovak Cultural and Educational Grant Agency (KEGA) under the Contract No. 062TUKE-4/2017.

\section{REFERENCES}

[1] PIETRIKOVÁ, A. et al.: "Construction of UWB Sensor System for M-Sequence Based on LTCC." Potential and services of USP Technicom for efficient development of entrepreneurship and research collaboration with industry. Košice: Elfa, 2015. 5357.

[2] RUMAN, K. - PIETRIKOVA, A. - GALAJDA, P. VEHEC, I. - ROVENSKY, T. - KMEC, M.: “A new approach to construction of extended kit for $\mathrm{M}$ Sequence UWB sensor system based on LTCC." Microelectronics International. Vol. 33, no. 3 (2016), p. 130-135.

[3] SACHS, J. - BADSTUBNER, A. - BONITZ, F. et al.: "High resolution non-destructive testing in civil engineering by ultrawideband pseudo-noise approaches." International Conference on UltraWideband, 2008, Hannover, Germany.

[4] LEVITAS, B.: "UWB time domain measurements." The Second European Conference on Antennas and Propagation, 2007. EuCAP 2007, pp. 1-8.

[5] LEVITAS, B. AND MATUZAS, J.: "Evaluation of UWB ISAR image resolution." European Radar Conference, 2005. EURAD 2005, pp. 89-91.

[6] DASCHNER, F. - KNOECHEL, R. - KENT, M.: "Rapid monitoring of selected food properties using microwave dielectric spectra." 2nd International
Conference on Microwave and MillimeterWave Technology, 2000, ICMMT 2000, pp. 670-673.

[7] HELBIG, M. - HEIN, M.A. - SCHWARZ, U. et al.: "Preliminary investigations of chest surface identification algorithms for breast cancer detection." International Conference on Ultra-Wideband, 2008, Hannover, Germany.

[8] PORCINO, D. - SACHS, J. - ZETIK, R. et al.: "UWB ranging." UWB Communication Systems a comprehensive Overview (eds M.G.d. Benedetto, T. Kaiser, A. Molisch et al.), 2006, Hindawi Publishing Corporation, pp. 411-446.

[9] OHTA, K. - ONO, K. - MATSUNAMI, I. et al.: "Wireless motion sensor using ultra-wideband impulse-radio." IEEE Radio and Wireless Symposium (RWS), 2010, pp. 13-16.

[10] HERRMANN, R. - SACHS, J. - SCHILLING, K. BONITZ, F.: "New extended M-sequence ultra wideband radar and its application to the disaggregation zone in salt rock." Proceedings of the 12th International Conference on Ground Penetrating Radar, GPR. Birmingham, UK, June 2008.

[11] SACHS, J.: Handbook of ultra-wideband shortrange sensing: theory, sensors, applications. John Wiley \& Sons, 2013.

[12] DANIELS, D. J.: Ground penetrating radar. John Wiley \& Sons, Inc., 2004.

[13] ROBENS, M. - WUNDERLICH, R. - HEINEN, S.: UWB LNAs for Ground Penetrating Radar. IEEE, 2009

[14] LIANG, H. - ZHIQIANG H.: "Study on signal processing of FMCW ground penetrating radar." Measuring Technology and Mechatronics Automation, 2009. ICMTMA'09. International Conference on. Vol. 1. IEEE, 2009.

[15] STICKLEY, G. F., et al.: "Gated stepped-frequency ground penetrating radar." Journal of Applied Geophysics 43.2-4 (2000): 259-269.

[16] PEČOVSKÝ, M., et al.: "Electrically short antenna with ground-coupling measurement for M-sequence ground penetrating radar." Progress in Electromagnetics Research Symposium-Fall (PIERSFALL), 2017. IEEE, 2017.

[17] GuidelineGeo. Mala GroundExplorer Ground Penetrating radar. http://www.guidelinegeo.com/wpcontent/uploads/2016/09/MALA-GX-TechnicalSpecifications.pdf.

[18] GuidelineGeo. Mala Mira 16 Ground Penetrating radar. Technical specifications. http://www.guidelinegeo.com/wpcontent/uploads/2017/10/Mala_MIRA_16_TechSpec _ForGeneralUse_FV.pdf.

[19] GSSI Antennas Brochure. http://www.alliedassociates.co.uk/wp-content/uploads/2016/03/GSSIAntennasBrochure.pdf. 
[20] PulseEKKO®. Sensoft, www.sensoft.ca/products/pulseekkopro/ specification/.

[21] Noggin $®$. Sensoft, www.sensoft.ca/products/noggin/specification/.

[22] PEREZ, R.: Handbook of Electromagnetic Compatibility. Academic Press, 2013.

[23] PEČOVSKÝ, M., et al.: "Budič krátkeho dipólu radaru na vyhl'adávanie objektov pod zemou na báze M-postupnosti.“ EEI 2017, Technical University of Košice, 2017.

[24] HARMUTH, H. F.: "Radiation of nonsinusoidal waves by a large-current radiator." IEEE transactions on electromagnetic compatibility 2 (1985): 77-87.

[25] HARMUTH, H. F. - MOHAMED, N. J.: "Largecurrent radiators." IEE Proceedings $H$ (Microwaves, Antennas and Propagation). Vol. 139. No. 4. IET Digital Library, 1992.

[26] Double Ridged Waveguide Horn - Model DRH200. [online]. [cit. 2016-04-12]. http://www.rfspin.cz/uploads/files/89f0a7078b ed0ecb32d81a052559f8b7593c09f3/drh200.pdf

Received April 16, 2018, accepted May 30, 2018

\section{BIOGRAPHIES}

Martin Pečovský was born in 1992 in Košice, Slovakia. He obtained his bachelor's and master's degrees with distinction in communication technologies in 2014 and 2016, respectively, from the Faculty of Electrical Engineering and Informatics, Technical University of Košice. He is currently a PhD. student at the Department of Electronics and Multimedia Communications. His current research focuses on analogue components of the $\mathrm{M}$ sequence radars, electrically short active antennas and RF circuit and chip design in international cooperation with Technische Universität Ilmenau, Germany and Ilmsens $\mathrm{GmbH}$.
Pavol Galajda was born in 1963 in Košice, Slovak Republic. He received the Ing. (M.Sc.) degree in electrical engineering from the FE TU in Košice and CSc. (PhD.) degree in radioelectronics from FEI TU in Košice, in 1986 and 1995, respectively. At present he is a professor at the Department of Electronics and Multimedia Communications, FEI TU in Košice. His research interest is in the design and implementation of Application-Specific Integrated Circuits (ASIC), the Ultra $\square$ Wideband (UWB) sensor and communication systems, the nonlinear communication systems and the systems generating Chaos.

Stanislav Slovák was born in Rožňava, Slovakia in 1989. In 2014 he finished MSc at the Technical University of Košice, Department of Electronics and Multimedia Communications, Faculty of Electrical Engineering and Informatics. He is now a PhD. student at the same department. His scientific research is mainly focused on research of application specific integration circuit for UWB applications.

Miroslav Sokol was born in 1993 in Vranov nad Topl'ou, Slovakia. He received master's degrees in Smartelectronics in 2017, from the Faculty of Electrical Engineering and Informatics, Technical University of Košice. He is currently a PhD. student at the Department of Electronics and Multimedia Communications. His research interest is in the design and implementation of Application-Specific Integrated Circuits (ASIC) for Ultra $\square$ Wideband (UWB) sensor and communication systems.

Martin Kmec received the Ing. degree in electronics and telecommunication technique from the Technical University Košice, Slovakia in 2000. In 2000 and 2001 he was with Meodat GmbH, Ilmenau, Germany, where his work has included the analysis and design of ultra-fast SiGe based integrated circuits. From 2001 till 2017, he was with Electronic Measurement laboratory on the Technical University Ilmenau in Germany as research assistant. His research interests are in the design, characterization and packaging of SiGe BiCMOS based circuits for novel UWB sensor systems. He is a co-founder of Ilmsens $\mathrm{GmbH}$, Germany, since 2016. 